\title{
The Effects of Adapting to Complex Motions: Position Invariance and Tuning to Spiral Motions
}

\author{
Robert J. Snowden and Alan B. Milne \\ University of Wales College of Cardiff
}

\begin{abstract}
Recent neurophysiological evidence (e.g., Graziano, Andersen, \& Snowden, 1994) suggested that some cells in the medial superior temporal area (MST) of the Old World monkey are sensitive to complex motions such as those brought about by a surface moving in depth or rotating. Two important findings were that these cells show position invariance (i.e., their preferred stimulus does not change across the receptive field), and that some cells were selective for "spiralling" stimuli rather than pure rotations or pure expansion/contractions. This paper attempts to provide evidence for similar processes in the human visual system by employing the technique of selective adaptation. We have simulated surfaces undergoing a motion in depth (div) or a rotation (curl), but have removed any cues that are not related to global motion. After adapting to a large pattern undergoing, say, an expansion, an aftereffect that contained an element of contraction could be elicited by placing small test patterns anywhere in the adapted area. This suggests
\end{abstract}

\section{INTRODUCTION}

Most studies of motion perception have concentrated on the simple linear motion of objects in the frontoparallel plane. The world, however, is not so obliging. The motion of objects may contain components toward or away from the observer; the object could rotate through a number of axes or even deform. Likewise movement of the observer can also produce complex motions across the retina. Gibson (1950) noted that the cues available to a pilot who is trying to land an airplane are extremely limited when static images are examined. Consideration of the dynamic properties of this scene can reveal, for example, that the scene expands from the direction the plane is heading, thus giving strong cues for pilots to exploit if they have the appropriate neural machinery to do so. Likewise substantial ecological advantage might be conferred to those individuals who can quickly and accurately determine their imminent collision (Lee, 1976) or that a baseball is heading toward their head (for a review, see Cumming, 1994). What is the evidence for the presence of such "complex" motion detectors?

that the global structure of the motion field must have been encoded as well as the local motion. Likewise thresholds for detecting motions similar to the adapting motion were elevated across the adapted area, while thresholds for other motions were not. Hence the effects of adaptation are both selective and show a degree of position invariance. Adaptation to pure div or pure curl stimuli was compared with adaptation to spiralling stimuli. Threshold elevation was always selective for the adapting motion and the shape and broadness of tuning did not vary. In simulations we could not reproduce our results using a model that had only div and curl detectors, but we could reproduce them if we allowed for detectors tuned for a broad range of spiral pitches. Our results suggest that humans encode the complex motion of surfaces by detectors tuned to many different types of motion and that the detectors are invariant across space in their properties.

The existence of cells sensitive to simple linear motions is abundant and established across a huge variety of creatures. In the primate, cells that are selective for particular directions of motion are found in striate cortex and many extrastriate areas, though they are absent from earlier parts of the visual system (for a review, see Snowden, 1994). One area has particular prominence for motion processing, the middle temporal (MT) area, as damage to this area blinds the animal to the motion of a stimulus while sparing other visual functions (Siegel \& Andersen, 1986; Newsome \& Paré, 1988). This area contains many cells that are sensitive to the direction of motion in the frontoparallel plane (Zeki, 1980; Albright, 1984; Snowden, Treue, \& Andersen, 1992) and to speed (Maunsell \& van Essen, 1983). Many MT cells send efferents to a nearby area, the medial superior temporal area (MST).A number of studies have shown that while some cells also respond most to frontoparallel motion, there are others that respond most to more complex motion of objects, such as rotations of the object or the object moving nearer or further away from the animal (Saito et al., 1986; Tanaka et al., 1986; Tanaka, Fukada, \& Saito, 
1989; Tanaka \& Saito, 1989; Duffy \& Wurtz, 1991a). Such responses remain even when considerable effort is made to remove any cues as to the motion that could be gleaned from nonmotion sources (Graziano et al., 1994). Hence it appears that in the Old World monkey there is indeed evidence for some complex motion detectors. What of humans?

A common psychophysical tool for demonstrating the existence of mechanisms in the human visual system has been that of selective adaptation (e.g., Mollon, 1974). For instance, the existence of elements in the human visual system sensitive to direction of motion is often inferred from the illusory motion one sees in the direction opposite to one that has been viewed for some time-the motion aftereffect (Addams, 1834; Wohlgemuth, 1911). The classic explanation for this phenomenon is that detectors tuned for the direction of motion that was being observed (let us say upward) become desensitized, thus creating an imbalance when the stationary pattern is viewed. This imbalance of activity (greater firing of downward detectors than upward detectors) normally occurs when there is some downward motion and, hence, the brain interprets these signals as the presence of downward motion.

Consider an experiment where a subject views a pattern whose elements are expanding away from the central point of the stimulus (this is often accompanied by a sensation of motion in depth toward the observer) and then fixates a stationary test target. Under such conditions a stationary test target may appear to contract (often accompanied by the sensation of motion in depth away from the observer). Such illusions of motion have been reported in several forms (Plateau, 1850; Regan \& Beverley, 1978a). Does this confirm the existence of detectors sensitive to expanding motions? By itself, no. Consider an area in the upper part of the adaptation pattern. Over this local area the motion will be nearly vertically upward. If one now considers a simple prediction from the model of motion aftereffects outlined above we predict a motion aftereffect in the opposing direction-downward. Applying the same argument to an area in the lower part of the adapting pattern we predict a upward aftereffect-and similar arguments can be applied to each part of the pattern. From this elementary analysis using only simple motion units the perceived aftereffect is predicted. Hence we do not need to invoke units specially designed to detect the expanding motion. In a similar manner Goldstein (1958) suggested that the spiral aftereffect (Holland, 1965) was merely the sum of a few normal motion aftereffects.

In a number of influential studies Regan and Beverley have provided evidence for the existence of detectors for complex motions by subtly altering the adaptation or test regimes. For example, Regan and Beverley (1978a) had subjects look at a square that changed in size, first getting larger then smaller in an oscillatory motion. They found that the minimum amplitude of motion required to detect this oscillation was drastically raised by previous viewing of a larger amplitude oscillation. The effect seems unable to be explained by simple motion aftereffects as thresholds for detecting leftward/rightward oscillations were little affected. It is the phase of the left and right motions (i.e., being out of phase) that is crucial to the large increase in thresholds, and therefore implicates the existence of detectors that are sensitive to the relative motion of the edges: complex motion detectors.

While Regan and Beverley's psychophysical demonstration is most convincing there are a number of limitations, not in the evidence for the existence of complex motion detectors, ${ }^{1}$ but in the application of this technique to other situations. The measurement of minimum amplitude thresholds does not allow for an analysis of faster speeds that we are more likely to encounter in real situations. The use of small squares may mean that minimum amplitude thresholds are detected by a position sensitive mechanism (Tyler \& Torres, 1972; Legge \& Campbell, 1981; Bonnet, 1982; Snowden, 1992), particularly for low rates of oscillation (Nakayama \& Tyler, 1981), rather than by a motion sensitive mechanism. We have therefore developed a stimulus that we believe eliminates such cues and will therefore isolate motion sensitive mechanisms. Our basic strategy is to use random dot patterns whose individual elements are displayed only briefly within the motion sequence. The use of random dot patterns to eliminate position-sensitive mechanisms has been demonstrated for minimum amplitude relative motion thresholds (Nakayama \& Tyler, 1981). Care has been taken with these stimuli to remove such cues as the changing size of the display or the elements within the display, the curvature or acceleration of element paths, or any changes in luminance (see section in Methods). Thresholds are then measured by degrading this stimulus with noise (see Methods), hence allowing us to measure thresholds at whatever speed we choose.

Having designed a stimulus that we believe will isolate the motion system and allow measurements to be made under a variety of stimulus conditions, how can we show that it is a genuine response to complex motions rather than the mere sum of linear components? We have modified a technique that has been employed by a number of physiological studies on the very similar question, namely, "how can we know that a cell is responding to the complex motion rather than just one bit of the motion contained within this stimulus?" One technique has been the "position invariance test" (Saito et al., 1986; Duffy \& Wurtz, 1991b; Graziano et al., 1994). In this test the response of a cell is tested with small stimuli that are positioned at several points within the cell's receptive field. For instance, Graziano et al. (1994) tested MSTd cells' selectivity for particular complex motions at five overlapping points in their receptive fields. Without exception each cell maintained the same preference (e.g., preferring expanding fields) at each point in the receptive field. Our technique uses a similar logic. We adapt 
the observer to a large field (for example, one that is expanding) and then test for aftereffects using small test patterns that can be placed anywhere within the field that had been adapted. Our logic is that if we have only simple linear motion detectors the observed aftereffect should follow from the motion that had occurred over the area that was subsequently tested. If, after we have adapted to a large expanding (movement toward the observer) stimulus, we test with a small stimulus positioned above the fixation point we should see a downward aftereffect, and if we test below the fixation mark we should see an upward aftereffect. If, however, we do have detectors tuned for complex motions then we may well see not upward or downward motion at these points but contracting motion (motion away from the observer).

The second aim of these experiments is to examine the notion of the range of complex motion detectors within the human visual system. Previous experimenters have provided evidence for expansion/contraction detectors (Regan \& Beverley, 1978a, 1978b) and for detectors for rotational motions (Regan \& Beverley, 1985). This evidence, along with findings of single cells within the primate brain sensitive to these stimuli, has been taken to support the notion that complex flow fields are analyzed into basis components. The suggested basis components are div (isotropic expansion/contraction), curl (rigid rotation), and def (deformation-expansion on one axis and contraction along the orthogonal axis), based upon the demonstration by Koenderink and colleagues (Koenderink \& van Doorn, 1975; Koenderink, 1986) that movement in any small region can be completely described by these differential invariants and a translational component (see also Longuet-Higgins \& Prazdny, 1980). Hence by simply having elements sensitive to just these dimensions the movement can be encoded. Such "basis type" schemes are common in vision (perhaps the most famous being the encoding of wavelength information by only three cone types in human vision). There are, however, other common schemes. For instance, in considering simple linear motion it is possible to encode the motion with just two basis functions, for example, one giving the vertical component and one the horizontal component of motion. We might, therefore, upon recording from directionally selective cells in a particular part of the brain expect to encounter only cells tuned to these axes. Indeed this is the case in some situations (the NOT in the rabbitSimpson, 1984). In area MT (see above) of the primate this does not appear to be the case. Instead we find a coding scheme such that there are cells tuned to all possible directions of motion with no bias for these axes (Maunsell \& van Essen, 1987; Albright, 1989). So it is possible that complex motions could be encoded by having cells tuned to each complex motion rather than just the basis functions.

Graziano et al. (1994) developed a scheme whereby motions such as expansions and rotations can be represented on two axes. They transform the motion of each from Cartesian to polar coordinates and then plot the radial speed on one axis (the ordinate) and angular speed on the other (the abscissa). Such a transformation is depicted in Figure 1. Hence an expansion is represented as having a positive div and no curl, whereas anticlockwise rotation has no div and negative curl. Directions in between these axes represent stimuli that spiral and have both div and curl components. We refer to this direction or angle as the pitch of the spiral. Graziano et al. (1994) have shown that in MSTd there are some neurons that do indeed respond maximally to these spiral stimuli rather than being just confined to the cardinal directions. Indeed, this study suggests that all possible pitches are represented with approximately equal weight (though some bias was found for expansions in general-see Discussion). Can we show a similar

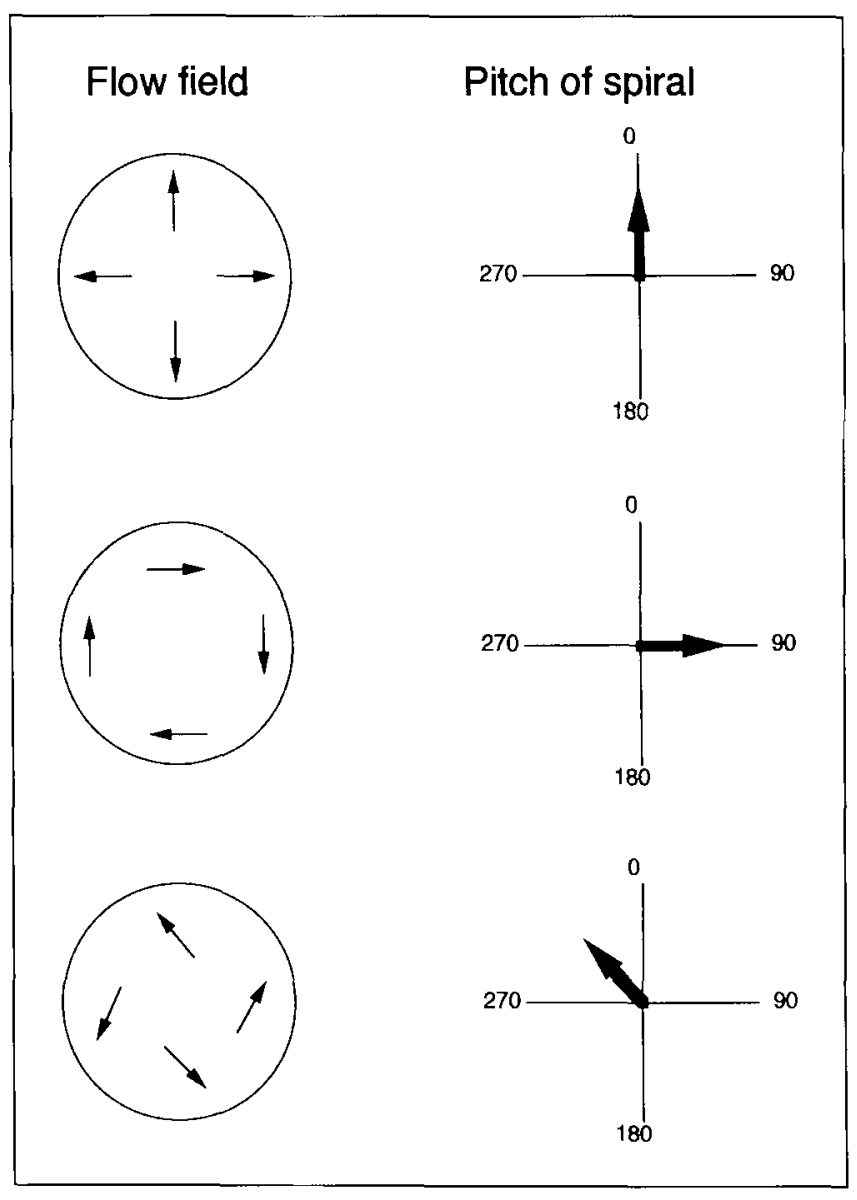

Figure 1. Representation of how the various flow field stimuli can be represented on a common frame of reference. The left side of the figure depicts three different flow fields, and the right side their polar coordinates are plotted with the ordinate representing the speed of the radial component and the abscissa the speed of the angular component. Uniform expansion corresponds to a positive radial value and no angular component and is represented with a pitch of $0^{\circ}$ (12 o'clock). Uniform clockwise rotation has no radial component and positive angular components and is represented by a pitch of $90^{\circ}$ (3 o'clock). A stimulus spiralling anticlockwise and expanding is represented with a pitch of $315^{\circ}$. 
situation in the human brain? To do so we have adapted subjects to various spiral pitches from both the cardinal directions and spiral stimuli from directions intermediate to the cardinal ones. We predict that if only the cardinal directions are represented in the brain we should only see peaks near these axes, whereas if there are elements tuned to a multiplicity of directions the peak of the adaptation function should lie at the point of adaptation.

Some of these results have already been presented in abstract form (Milne \& Snowden, 1993; Snowden \& Milne, 1994).

\section{RESULTS}

\section{Informal Observations}

We have performed a number of demonstrations/observations of an informal nature (i.e., no quantitative results have been collected). The observations are mainly those of one of the authors (R. J. S.), but the crucial ones have been shown to various friends and colleagues. Unless otherwise stated all were able to verify the observations described below.

Our first observation was to check that we could elicit motion aftereffects from our stimuli. We adapted to various different patterns such as rotations, expansions, and contractions and tested with a large stationary dot pattern. In all cases we observed the expected aftereffects-adaptation to clockwise rotation produced illusory rotation in the opposite direction, adaptation to expansion produced an illusory contraction, etc. We then attempted to test for a position invariance of this aftereffect. The procedure behind the experiment is outlined in Figure 2B. We once again adapted to a large pattern but then tested with small patterns placed in turn at each of the five positions illustrated in Figure 2B. Our rational was to see whether, if when the adapting pattern was, say, an expanding flow field, the aftereffect would follow from a consideration of the motion that had taken place over the test site, or whether the global motion

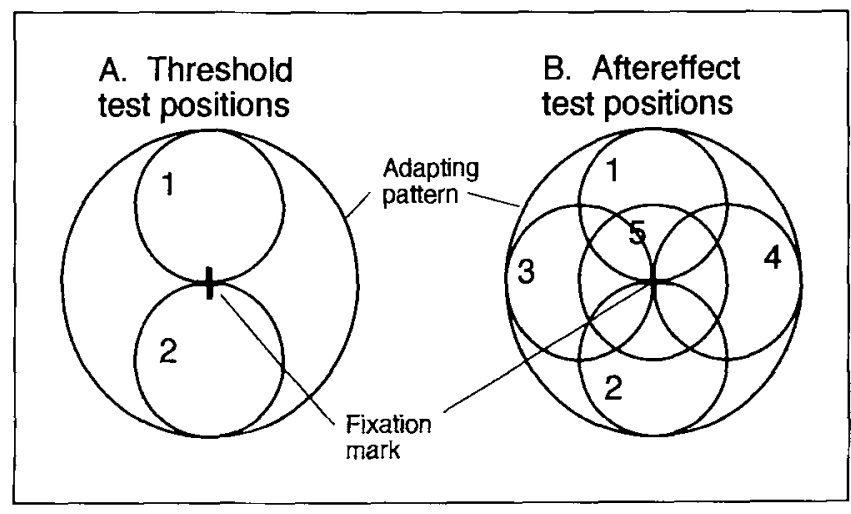

Figure 2. Schematic representation of the adapting and testing positions used for the position invariance tests. The larger circles represent the size of the adapting pattern, while the smaller circles represent the size and position of the test patterns. would be expressed in the aftereffect. After adaptation to either div stimuli the aftereffect clearly had components of both the global and local configurations. In position one, after adaptation to an expansion, the test pattern appeared to move away from the observer and slide slightly downward, whereas at position 2 it appeared to move away and slide upward, etc. We shall term the movement in depth the "global component" and the linear sliding the "local component." Under the conditions described in the Methods section the global component was most noticeable. During pilot trials we tried several ways to maximize this component. Though no formal trials have been conducted several observations seem worth mentioning. The global component was quite ephemeral. Increases in the dot density of the test pattern, for example, seemed to reduce the global component (or alternatively increase the local component) so that only the local component was visible. The global effect was much stronger in the central position than in any other position. This is not surprising as any linear components would reinforce the effect in this position. The global component seemed stronger in the upper position (1) rather than the lower position (2). Experiments where we fixated just outside the area of the adaptation pattern seemed to produce a greater global component. The adapting pattern did not always appear to move in depth; sometimes it simply appeared as an expansion without any perceptual feeling of depth change. The global component of the aftereffect seemed stronger when it immediately followed a period where the adapting pattern gave the phenomenon of motion in depth.

After adapting to curl stimuli similar position invariance tests were performed. Once again both global and local components were visible. The test stimuli at positions 1-4 appeared to rotate a little and slide in a direction befitting the conventional motion aftereffect. This observation, however, was not confirmed by all observers. About half of our observers agreed with this observation, while others reported a less vivid global effect than in the div case. Two observers did not report any global component. Interestingly these two observers who did not report the global component after the curl stimuli did report the global component after the div stimuli. This, therefore, appears to be a difference in the stimulation rather than an individual difference in reporting the aftereffects.

Aftereffects of div and curl have been well documented and were also found in this study. We also adapted to various spiral stimuli. The aftereffect did indeed spiral in a predictable manner, for example, if we adapted at $315^{\circ}$ (see Fig. 1) we produced an aftereffect that resembled a $135^{\circ}$ stimulus. Note this spiralling aftereffect ${ }^{2}$ is different from the spiral aftereffect, which refers to the effects of adapting to stimulus that is spatially a spiral undergoing rotation (Holland, 1965). We also performed the position invariance test for our spiral 
stimuli. Once again a convincing spiral of opposite polarity was produced at each of the test positions.

\section{Threshold Measurements}

\section{Position Invariance Tests}

We measured thresholds for detecting expansion or contraction at two positions in the visual field (just above and just below the fixation point) after adapting to a large pattern (see Fig. 2A). Figure 3 shows the results for one subject. The upper panel represents the results for detecting the expansion stimulus whereas the lower is for the contracting stimulus. Within each panel results are shown for when the test stimulus was in the top position (the left half) and the bottom position (the right half). The different bars represent the various adapting conditions - a blank field (filled bars), a contracting field (stippled bars), or an expanding field (open bars). The pattern of results shows that prolonged viewing of an expanding flow field elevates thresholds for detecting an expanding flow field over its baseline (where the adaptation pattern was blank) but leaves the detection of the contracting flow field unchanged. Prolonged viewing of a contracting flow field, on the other hand, elevates thresholds for detecting contracting flow fields but leaves the detection of expansion unaffected. This was true for both positions in the visual field. Figure 5 (upper sections) plots results of the same experiment for a
Figure 3. Thresholds are plotted for detecting an expanding field (upper section) or a contracting field (lower section). The position of the test stimulus (top or bottom of the fixation mark) is shown on the abscissa. The type of adapting field is represented by the different bars; solid is for no adaptation, stippled for adaptation to contraction, and unfilled for adaptation to expanding fields. Error bars represent 1 SEM; the subjects was EJ.

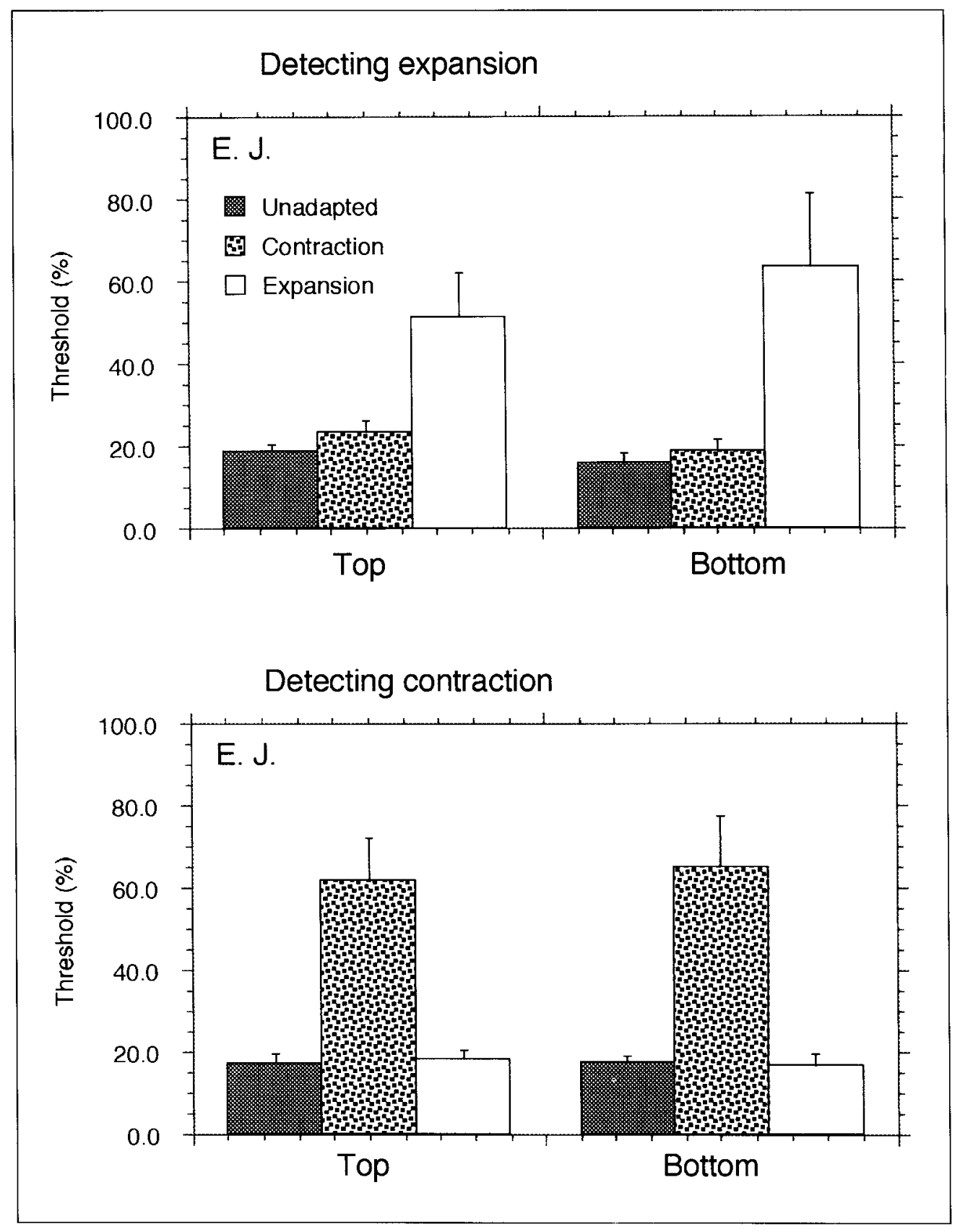


second observer. The results here are plotted as the more conventional threshold elevation plots [the ratio of adapted to unadapted thresholds in decibels $(20 \mathrm{~dB}=1$ $\log$ unit)]. Again the results show that prolonged viewing of one type of flow field elevates thresholds for that type of motion, but leaves the other unaffected, in both test positions.

Results from similar experiments where subjects were adapted and tested with rotations are shown in Figures 4 and 5 (lower sections). The results show that adapting to a clockwise rotation elevates thresholds for clockwise rotation but does not affect thresholds for anticlockwise rotation (the decrease in thresholds seen under some conditions never reached significance on a Student's $t$ test). In a similar vein adapting to an anticlockwise rotation elevated thresholds for anticlockwise rotations but left thresholds for clockwise rotations unchanged. Again this pattern of results is consistent across positions. It worth noting that the scale on Figure 4 is different to that of Figure 3. This subject (EJ) showed markedly less threshold elevation in the experiments using curl stimuli than she did in those using div stimuli. For the subject whose results are displayed in Figure 6 we see a similar magnitude of threshold elevation in all experiments. Interestingly subject EJ did not report position invariant curl aftereffects, but did report position invariant div aftereffects, whereas subject RS reports the position invariant aftereffects in both conditions.

These results therefore show that adapting to a particular type of complex motion increases thresholds for detecting that motion and that the increase in thresholds is not confined to patterns whose central point coin-
Figure 4. As for Figure 3 but now for clockwise and anticlockwise stimuli. Note the change of scale on the ordinate.

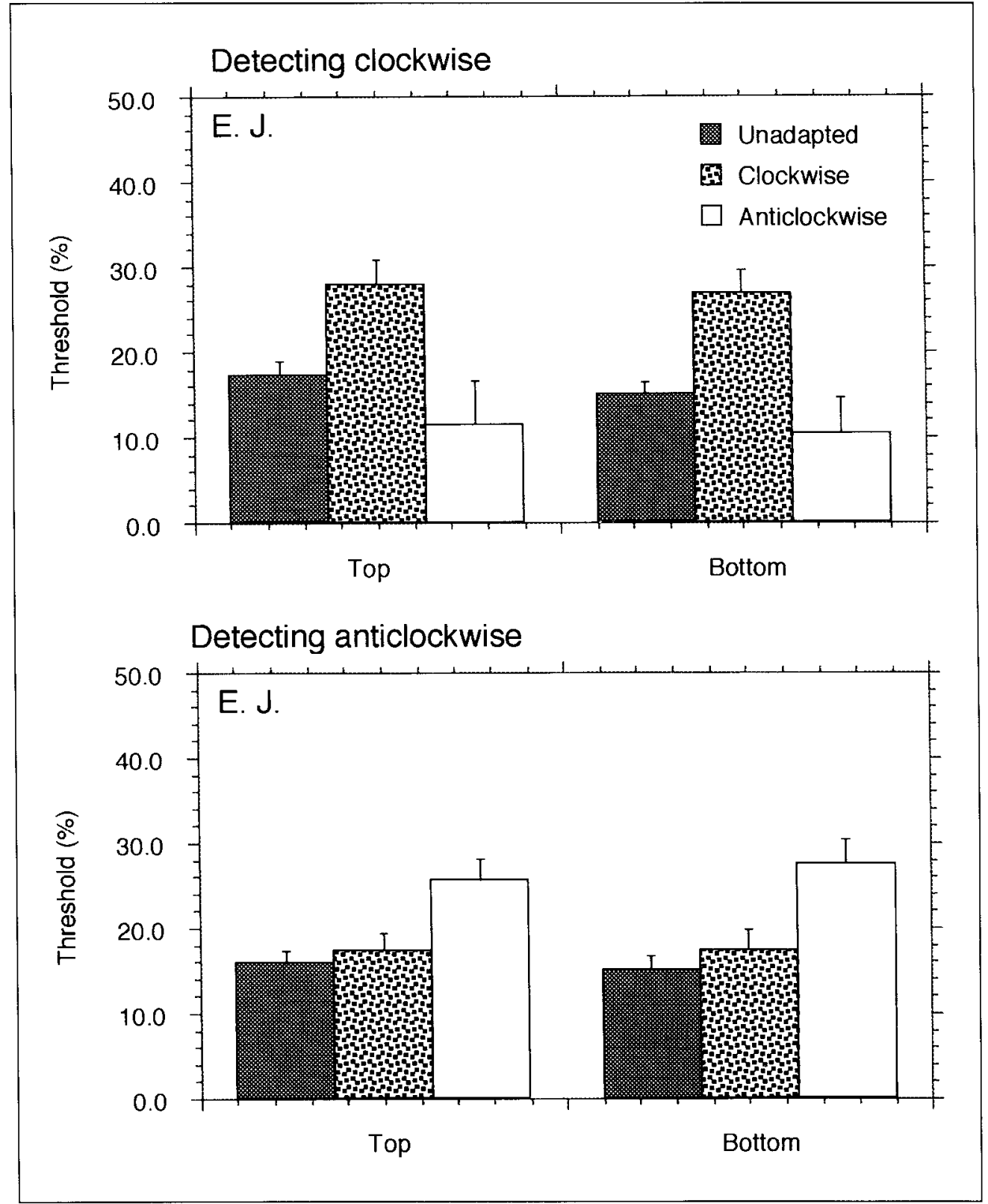


Figure 5. Threshold elevation (ratio of adapted to unadapted thresholds) is plotted for the div stimuli (upper panels) and curl stimuli (lower panels). The different bars represent different adapting patterns. The subject was RS.

\section{Adapting to}

圈 Contraction

Expansion
Detecting expansion

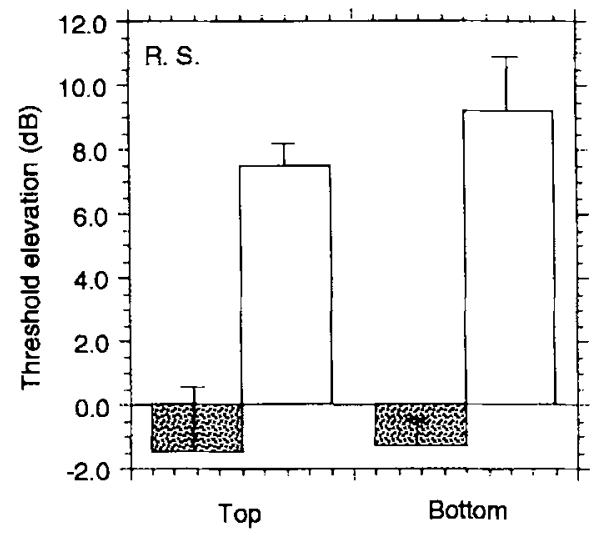

Detecting contraction

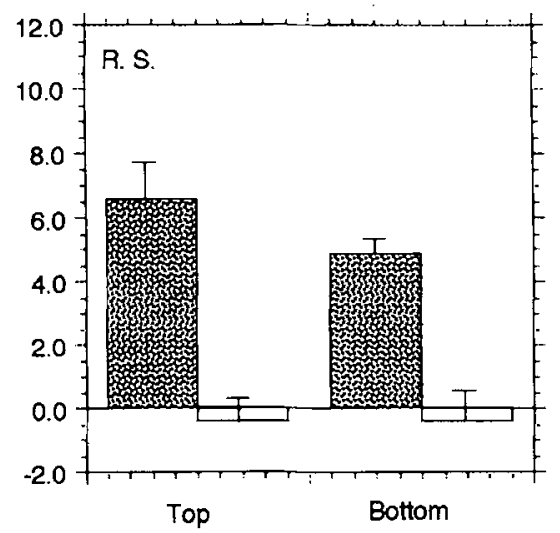

Adapting to

$\square$ Clockwise

圆 Anticlockwise

Detecting clockwise

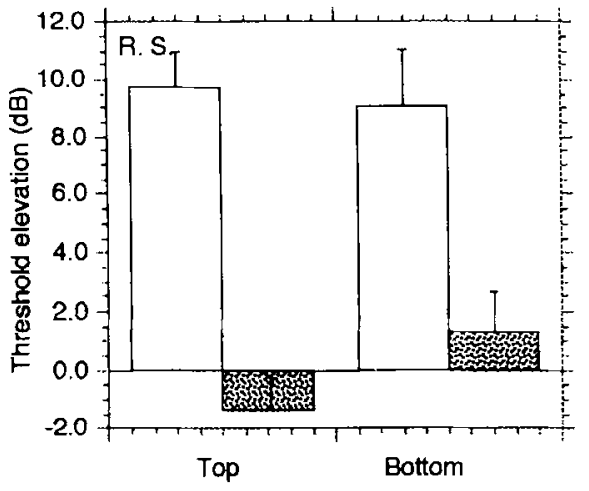

Detecting anticlockwise

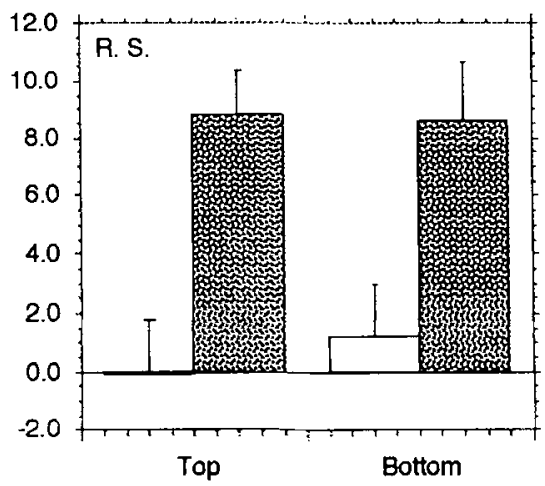

cides with the center of the flow field. There is at least a degree of position invariance to the threshold elevation effect.

\section{Baseline Sensitivity}

Having established the threshold elevation effect and its position invariant qualities we now attempted to look at its specificity and whether such adaptation effects occur only for the postulated differential invariants (div and curl) or could be induced for any arbitrary spiral stimulus. We therefore began by measuring thresholds for stimuli in the absence of any adapting pattern. These results are shown in Figure 6, where we plot the threshold correlation as a function of spiral pitch. The symbols represent data from the individual observers whereas the dotted thick line represents the average. Sensitivity to these stimuli varied somewhat from observer to ob- server, from average thresholds of approximately $13 \%$ to approximately $7.5 \%$. Overall sensitivity to the various patterns was approximately similar for all stimuli, though there are some small trends in this data. Subjects tended to be more sensitive to the pure curls than any other stimuli. In addition, subjects tended to be more sensitive to stimuli containing a component of contraction than one of expansion. The latter trend is in line with a recent report using similar techniques (Edwards \& Badcock, 1993). ${ }^{3}$

\section{Adaptation to Spirals}

We now remeasured these thresholds after adapting to various flow stimuli. Figure $7 \mathrm{~A}$ shows the results for one subject (RS) after adapting to a clockwise rotation $\left(90^{\circ}\right)$. The inset to the figure plots the baseline threshold (open symbols) and the experimental thresholds (filled sym- 
Figure 6. Thresholds for detecting the test patterns in the absence of adaptation. The open symbols represent the data of five individual subjects and the dotted thick line is the average of these subjects.

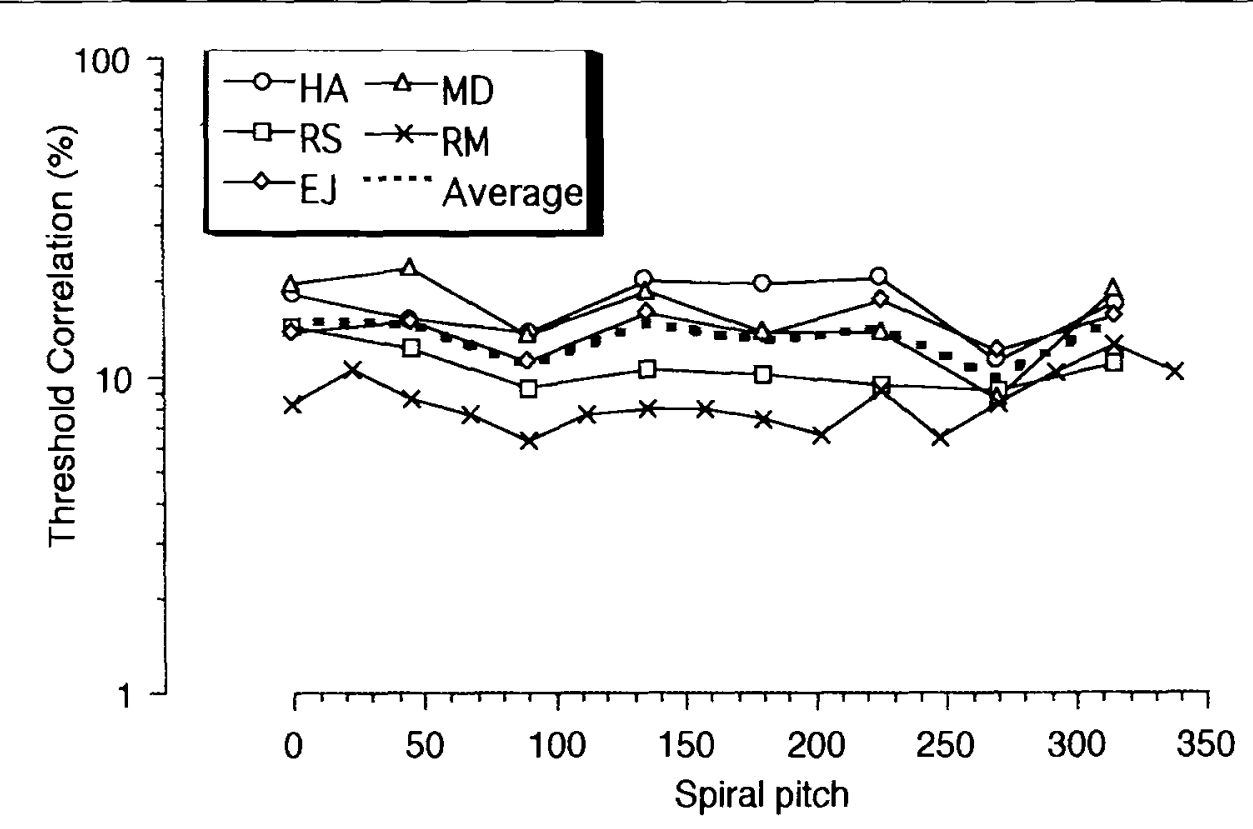

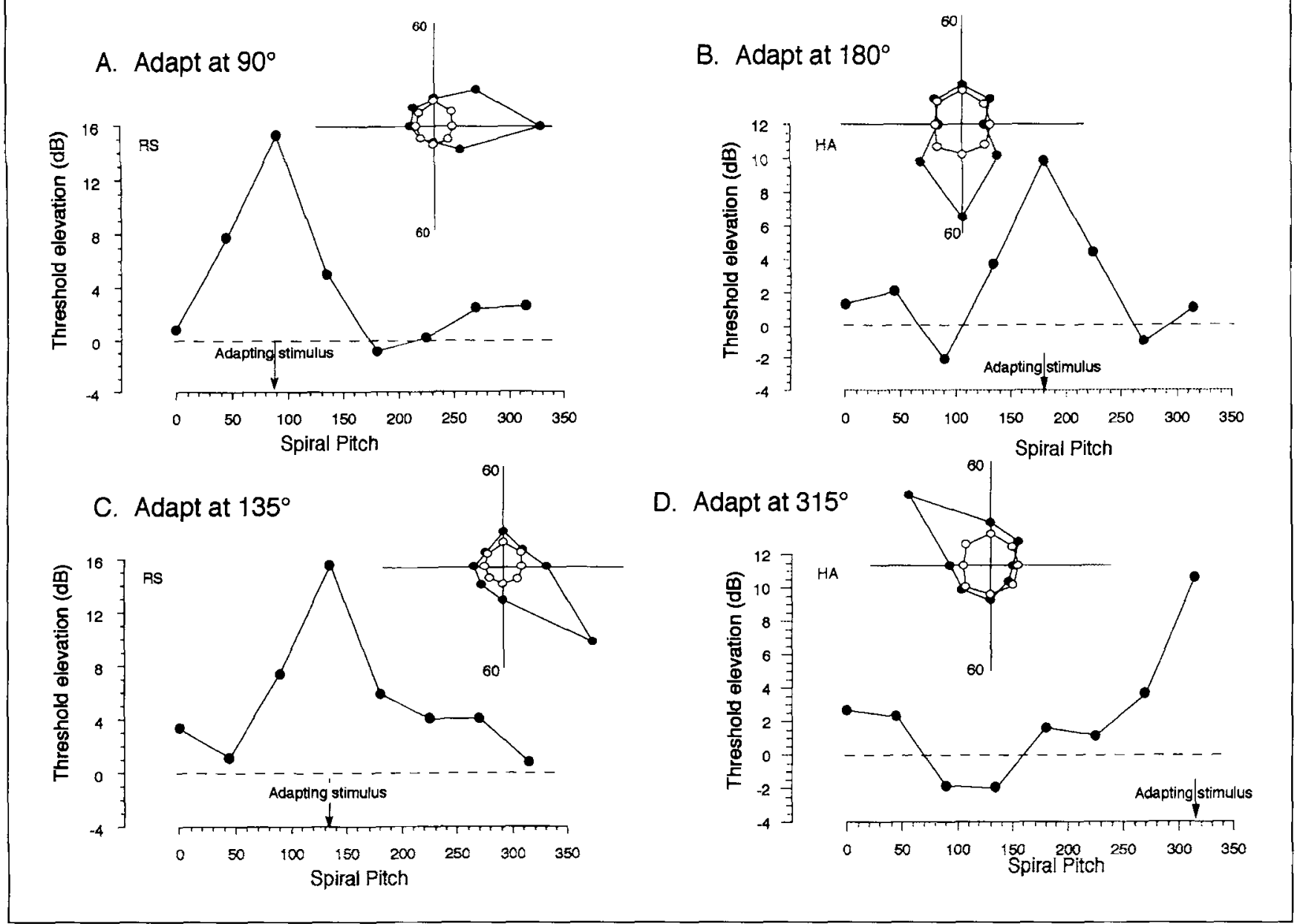

Figure 7. The effects of adaptation are plotted as a function of the pitch of the test pattern. In the main plots threshold elevation is plotted (ratio adapted/unadapted threshold in decibels). In the insets the actual thresholds are plotted in the pitch space represented in Figure 1. (A, B) Cardinal directions; (C, D) noncardinal directions. 
bols) in the coordinate system of Figure 1 with the center of the graph representing a threshold of $0 \%$, and the end of each axis a threshold of $60 \%$. The main plot represents the threshold elevation for each test stimulus. As found previously, prolonged viewing of a clockwise rotation elevates threshold, in this case from approximately $10 \%$ to approximately $50 \%$-or a $15 \mathrm{~dB}$ increase in threshold, but does not affect thresholds for anticlockwise rotation. Further, we can see that the clockwise rotation does not have any effect upon thresholds for detecting expansion or for detecting contractions (i.e., for patterns $90^{\circ}$ apart in this coordinate system). Patterns $45^{\circ}$ away from the angle of adaptation are somewhat affected. A similar pattern of results was found after another subject adapted to a contracting stimulus (HA$180^{\circ}$ ) and is displayed in Figure $7 \mathrm{~B}$. These measurements confirm that the adaptation effect is greatest for pattern of the same motion characteristics, and quickly falls off when the adaptation and test patterns differ.

The results of adapting to spiralling stimuli are shown in Figures $7 \mathrm{C}\left(\mathrm{RS}, 135^{\circ}\right)$ and $7 \mathrm{D}\left(\mathrm{HA}-315^{\circ}\right)$. The results resemble those found when adapting to rotating or contracting stimuli in two important respects. First, the threshold elevation function is markedly peaked, and this peak occurs at the adapting direction. Second, the threshold elevation has the same tightness of tuning as for the cardinal directions. Results from a third subject who adapted to two cardinal and two noncardinal pitches are shown in Figure 8. As a measure of this tuning Gaussian functions were fit to the threshold elevation data. Table 1 gives the results of these fits. The standard deviation of the best fitting Gaussian (hereafter termed bandwidth) is on the order of $30-40^{\circ}$.

\section{Simulation}

The results of the previous section point to the notion that stimuli from noncardinal directions are processed in a manner similar to those from cardinal directions. We therefore suggest that there may be a range of detectors (filters) tuned to all (or many) directions of this space rather than just the cardinal directions. We have run simulations using either just four-filters (tuned to the cardinal directions), and many filters (termed multifilter) to see if the four-filter simulation could, in principle, still produce results similar to those described above. The simulations consisted of a number of filters of Gaussian profile. The output of each filter is passed through a correlation-response function whose characteristics can be altered by adaptation. The amount of adaptation on any filter is governed by its strength of response to the adapting stimulus. Threshold for detection is taken as the point when any filter reaches a level of activation above a set (arbitrary) level. Clearly this simulation required that a number of assumptions be made-the shape of the filters, the definition and level of threshold, the nature of the correlation-response function, how adapta-

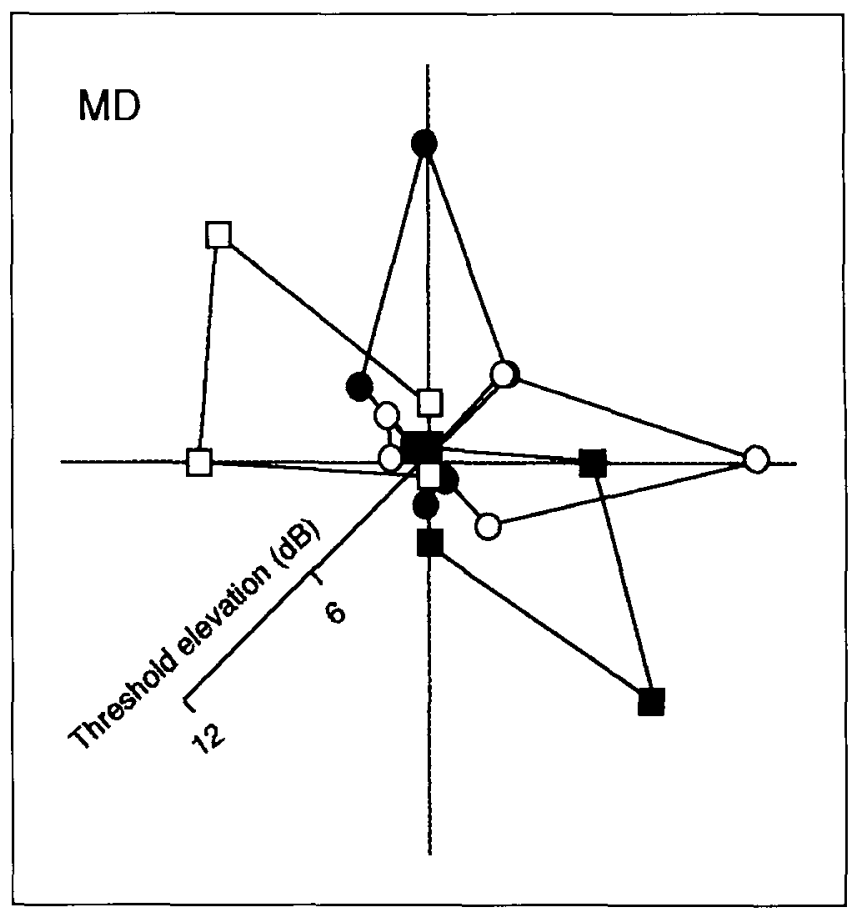

Figure 8. Threshold elevation is plotted in pitch space for subject MD after adapting to two cardinal directions $\left(0^{\circ}\right.$ - filled circles, $90^{\circ}-$ open circles) and two noncardinal directions ( $135^{\circ}-$ filled squares, $315^{\circ}$-open squares). Data points that were negative have been omitted for clarity.

Table 1. Gaussian Fits to Adaptation Data

\begin{tabular}{rcrcc}
\hline & Height & Peak & Bandwidth & $R$ \\
\hline 0-MD & 11.4 & 2 & 30 & 0.97 \\
$90-$ MD & 12.8 & 88 & 28 & 0.94 \\
$90-$ RS & 14.8 & 84 & 33 & 0.96 \\
135-MD & 12.6 & 126 & 30 & 0.96 \\
135-RS & 15.5 & 134 & 39 & 0.86 \\
$180-$ HA & 10.1 & 182 & 32 & 0.91 \\
$315-$ MD & 13.6 & 302 & 29 & 0.98 \\
$315-$ HA & 10.5 & 312 & 30 & 0.92 \\
\hline
\end{tabular}

tion affects the model, and leaves us free to vary other parameters such as the width of the filter, the size of the adaptation effect, etc. The appendix describes the simulation in detail and our justification of the decisions we took. We do not want to put this simulation forward as an accurate model; we were merely seeking to see if either the four-filter simulation or the multifilter simulation could produce a reasonable representation of our results.

The four-filter model had a number of difficulties in reproducing our results. First, our baseline results suggest that we are approximately equally sensitive to all our stimuli. If we had filters of a narrow bandwidth 
$\left(<50^{\circ}-\right.$ bandwidth here is defined as the standard deviation of the Gaussian) then we found sensitivity was markedly reduced for the spiral stimuli in comparison to the div or curl stimuli. To illustrate this we compared sensitivity at the cardinal direction with that of pitches halfway between the axis. Figure 9 shows the ratio of sensitivities as a function of the filter bandwidth. We found that by increasing the bandwidth to $70^{\circ}$ we still had approximately $10-20 \%$ falloff in sensitivity for the spirals in comparison to the cardinal directions, but that our accuracy of measurement was not sufficient to rule out this possibility. Greater bandwidths did produce more uniform sensitivity. Our simulations of adapting bandwidth for both a cardinal direction and a noncardinal one are also shown in Figure 9 as a function of filter bandwidth. From Table 1 we see that the psychophysical data suggest adaptation bandwidths on the order of $30^{\circ}$. Such narrow bandwidths were never produced by this model when adapting to a noncardinal direction, and we could not find a filter bandwidth that could produce near uniform sensitivity to all pitches and adaptation bandwidths on the order of $30^{\circ}$. When we then adapted the model $\left(70^{\circ}\right.$ filter) we found that the shape of the adaptation function was different for cardinal and noncardinal directions. The adaptation function became flatter and did not resemble the psychophysical data (see Fig. 10). Thus the four-filter model failed to produce results that resembled several aspects of the psychophysical data.

The multifilter simulations consisted of 18 filters spaced at intervals of $20^{\circ}$. We used a bandwidth of $40^{\circ}$ as this seemed consistent with what little physiological evidence is available (Graziano et al., 1994). Before adaptation this simulation produced near uniform sensitivity for each direction (maximum difference ratio $=1.04$ ). The threshold elevation functions after adaptation were
Figure 9. Results from the four-filter simulation of our exbetween stimuli cardinal and noncardinal directions is plotted in the filled symbols (see right-hand ordinate). The bandwidth of adaptation for a cardinal $\left(180^{\circ}\right.$-open circles) and a noncardinal direction $\left(135^{\circ}-\right.$ open squares) is plotted as a function of simulation filter width on the left-hand ordinate. periments. The sensitivity ratio

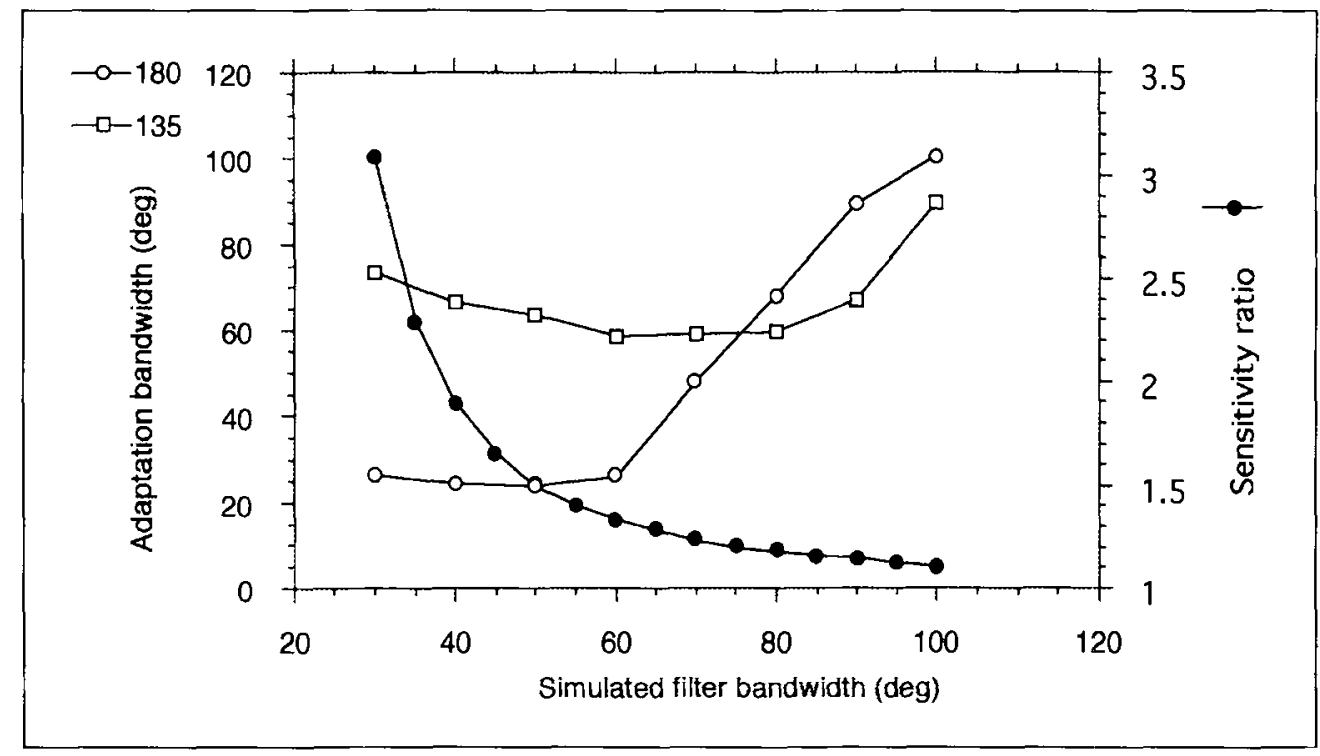

Figure 10. Representative results from the simulations involving four filters (A) or multifilters (B). The plots are as for Figures 7 and 8 save that the filled symbols are for adapting to $270^{\circ}$ and the open symbols are after adapting to $225^{\circ}$ (not unadapted).

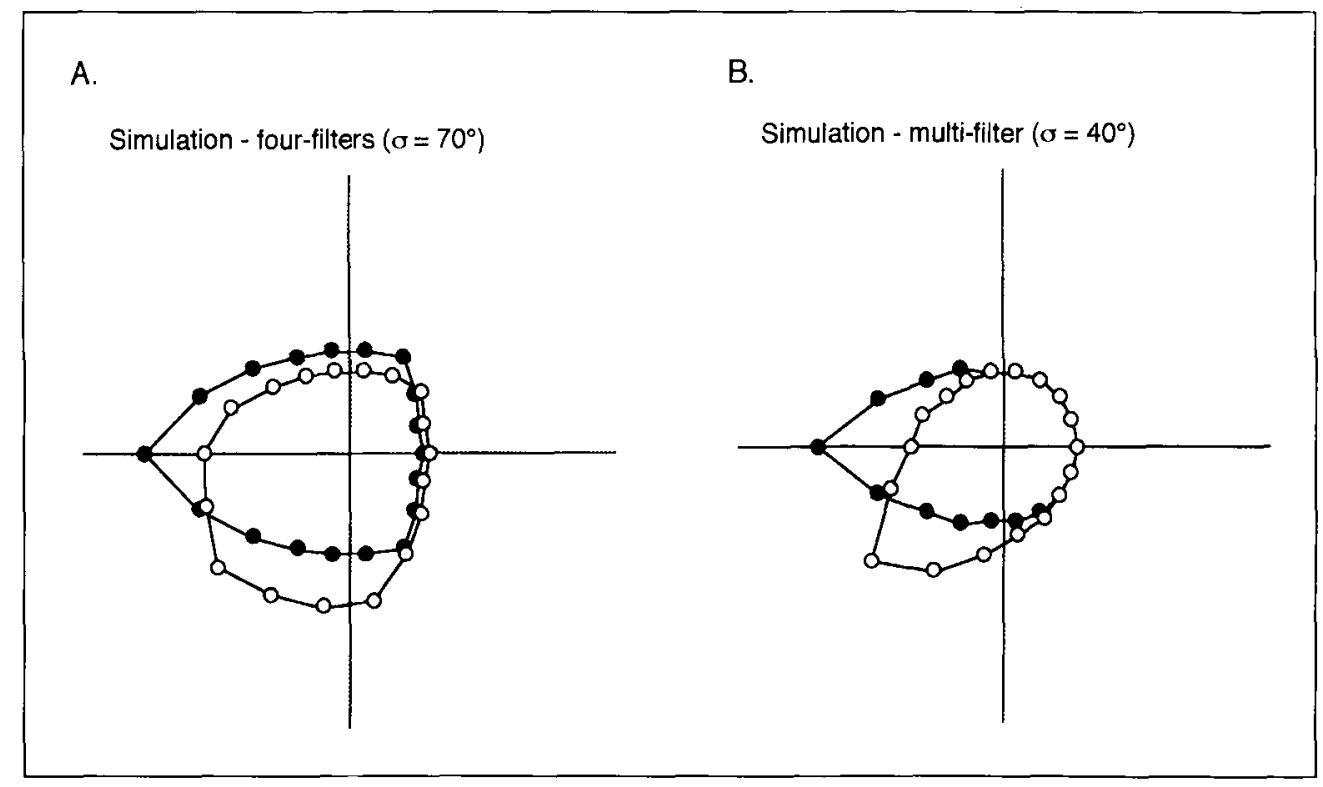


similar in shape and form for stimuli from both the cardinal directions and the spirals (see Fig. 10). The bandwidths for these threshold elevation functions were both approximately $30^{\circ}$. Hence the multifilter version was able to simulate the psychophysical results with no further assumptions.

\section{DISCUSSION}

Our results point to two conclusions, namely that the human visual system has specialist detectors for complex motions and that these detectors are not confined to those predicted by the notion of differential invariants. We shall discuss our evidence for these conclusions in light of other psychophysical evidence and relate it to our current understanding of the physiology of motion perception.

\section{Evidence for Complex Motion Detectors}

In the introduction we have already mentioned evidence from studies by Regan and colleagues that suggest mechanisms sensitive to div (Regan \& Beverley, 1978a) and curl (Regan \& Beverley, 1985) using adaptation techniques. Further evidence for curl detectors is provided by Cavanagh and Favreau (1980). They adapted subjects to a rotating logarithmic spiral and then tested with a similar spiral or one that was the mirror image of the adapting spiral. For spirals that are the mirror image of one another all contours are at $90^{\circ}$. The motion aftereffect was observed in both cases, but was somewhat less for the mirror image stimulus. They argue that the aftereffect obtained for the mirror image spiral must be indicative of a global mechanism, as our current understanding of linear motion detectors suggests they would be insensitive to contours that differ by $90^{\circ}$. They further show that the storage properties of the "global" mechanism are somewhat different to the normal motion aftereffect-a point also made by Regan and Beverley (1978b; though also see Hershenson, 1993). Thus Cavanagh and Favreau (1980) suggest that these stimuli elicit both a motion aftereffect explicable in terms of the local motion at each point in the adapting stimulus and a more global effect. This is very similar to the results found in the present study. Neither this study nor the present one has so far quantified the conditions that will maximize either the local or the global components. This would be a most useful step for future investigators. Further recent evidence (Culham \& Cavanagh, 1995) suggests that "global" aftereffects and "local" ones may be mediated by both the nature of the test stimulus (flickering versus static) and whether the subject attentively tracks the adapting motion (tracking producing a greater global component).

A second finding that points to global motion detectors comes from the finding of phantom motion aftereffects. For instance, Bonnet and Pouthas (1972) found that after adapting to four small sections of a rotating spiral the aftereffect was not confined to the small sections but could spread to the whole of the spiral. While phantom motion aftereffects have been observed in some special circumstances for linear motions (Weisstein, Maguire, \& Berbaum, 1977) it seems hard to understand the phantom spiral aftereffect in such terms. The results seem to suggest that the small sections are enough to activate and adapt a complex motion detector. As the hypothetical complex motion detector would have to have a large receptive field to integrate the motion from the various sections, its influence is felt across the receptive field area and hence even in the sections that were not directly stimulated by the adapting pattern(s).

A recent study (Morrone, Burr, \& Vaina, 1995) provides further evidence for the existence of specialist motion detectors with large integration fields. They found that spatial summation of motion signals in stimuli somewhat similar to the present study could take place across different sectors of the pattern (of course the motion in the different sectors is quite different) implying a mechanism whose motion preference varies with its position. Contrast sensitivity for these complex motions, however, did not greatly increase with increasing number of sectors implying that the specialized detectors are preceded by an earlier stage of local motion mechanisms that impose a contrast threshold.

\section{Basis Functions}

Several authors have examined human sensitivity to optic flow type stimuli. Of particular interest here are the findings of Freeman and Harris (1992). They show that the minimum amplitude of motion is smaller for div and curl stimuli than for the same patterns with the directional vectors of each element randomly rotated (somewhat like the current experiments), or those for translating stimuli of equal speed profiles. They argue that this enhanced sensitivity must be due to detectors that are sensitive to the spatial arrangement of the motion vectors-complex motion detectors. A second aspect of interest to the present study was the small but consistently greater sensitivity to the curl stimuli than to the div stimuli. This was also apparent in the present data using the signal-to-noise ratio paradigm.

One of the major aims of these experiments was to examine the validity of the idea that humans decompose optic flow fields into differential invariants, i.e., do we have detectors only for div and curl. The present results seem to reject this notion. This theory would predict that subjects should be most sensitive to these particular patterns and also predicts that any adaptation effects would be the sum of the adaptation caused in the div and curl filters and would therefore be different when the stimulus was actually a pure div or curl than when it was a hybrid of these two stimuli. The results do not 
appear to support these predictions. While our simulations with just four filters corresponding to the div and curls failed to produce a pattern of results that corresponded to those found psychophysically, we did find that a multifilter model could produce results very similar to the human data. Other psychophysical results impinging on this issue are sparse, presumably because other experimenters did not consider the possible range of stimuli that can be conceptualized if one considers these patterns in a common representation space.

The bandwidth of elevation in our coordinate system was on the order of $30^{\circ}$ (and was modeled by filters having a bandwidth of approximately $40^{\circ}$ ). This implies that div and curl detectors (or more specifically any two patterns $90^{\circ}$ apart on our axes) have little overlap in their tuning functions and therefore should show little interference. Again the results of Freeman and Harris (1992) seem to support this notion. They show that adding a div mask stimulus to a curl test stimulus had little effect on test thresholds; likewise adding a curl mask to a div test had little effect. This also implies near independence of detectors for these stimuli (see also De Bruyn \& Orban, 1993).

\section{Relationship to Physiology}

The present experiments were prompted by the findings from cells within the medial superior temporal area of the macaque monkey. Some of these cells are sensitive to div or curl stimuli. Recently Graziano et al. (1994) have also shown that there exists cells whose preference is not for pure div or curl but for something in between-spiralling stimuli. Our present psychophysical results complement these findings by demonstrating threshold elevation functions after adaptation that are tuned for the pitch of the adapting stimuli, be it from one of the cardinal directions or not. Though no comprehensive data yet exist for the tuning characteristics of the MST cells, the few cells reported in the Graziano et al. study have bandwidths on the order of $40^{\circ}$. These cells therefore seem to have the necessary properties to make them candidates for the neurophysiological substrate of the present effects. Several studies have demonstrated the position invariance of the response of MST cells to these complex motions (Saito et al., 1986; Tanaka et al., 1986, 1989; Tanaka \& Saito, 1989; Duffy \& Wurtz, 1991b; Graziano et al., 1994; though also see Orban, Lagae, Raiguel, Xiao, \& Maes, 1995) with the study of Graziano et al. (1994) confirming this for spiral stimuli as well. If one considers the possibility that such cells will be adapted due to the large adapting stimulus, then this may make it harder to detect the small stimuli of similar motion characteristics wherever they appear within this field. The motion aftereffect could then be due to a "distribution shift" in the overall response of area MST in a manner analogous to that suggested for the conventional motion aftereffect (Mather, 1980).

While the above paragraph attempts to draw attention to the similarities of the properties of cells in area MST and the current results there are discrepancies worth noting. Graziano et al. (1994) found more cells with preferences for components of expansion than for components of contraction (e.g., a centrifugal bias), or to curl. This is the opposite of the present results, which show subjects to be most sensitive to curls, then contraction and finally expansion. A somewhat analogous situation occurs in considering linear motion. Albright (1989) has shown that there is a bias in the preferences of MT cells such that there are more cells that prefer an element of centrifugal motion than there are ones that prefer an element of centripetal motion (which may, in turn, explain the centrifugal bias in MST). However, measurements of human subjects' sensitivity to linear motion using techniques similar to those presented here show a centripetal bias (Raymond, 1994), which may, in turn, explain a human centripetal bias in the case of complex motions (Edwards \& Badcock, 1993-see also note 3). The reason for all these discrepancies is unclear. They may be a species difference (the physiological data have been gathered from macaques, whereas the psychophysical data have been gathered from humans). It may be that while there may be more cells with a centrifugal bias, these cells are less sensitive and do not show this bias when threshold measurements are taken. Interestingly some suprathreshold measures of human performance (i.e., reaction times) show a centrifugal bias (Ball \& Sekuler, 1980). Clearly this particular point needs further investigation.

\section{Global versus Local Processes}

Our results point to the existence of processes that are sensitive to the relationship between velocities (or directions) within a stimulus. This may be thought of as a global mechanism that in turn changes the perception of a local part of the image. Thus an upward motion in part of our stimuli is not necessarily interpreted as upward motion, but may be seen as part of an object/surface moving toward the observer. Other researchers have also been looking for the influence of global processes on aftereffects. Emerson, Humphrey, and Dodwell (1985) used a variation of the contingent aftereffect paradigm. Subjects adapted to red concentric circles (i.e., bull's-eyes) and green radial patterns (spokes), then red vertical gratings and green horizontal gratings. Upon testing they found that the aftereffect was governed by the global configuration with no interference from the local configuration. For example, if presented with an achromatic bull'seye it would appear tinged with green even though some of the bull's-eye's contours are near horizontal and these had been paired with green and thus should produce a red aftereffect if the local configu- 
ration is important. However, there are reports against such a notion (McCollough, 1994), including one using moving stimuli (Broerse, Shaw, Dodwell, \& Muir, 1994). While these latter studies suggest that the local configuration helps to determine the aftereffect, they do not rule out the notion that there is a global aftereffect that is also present. In relation to the present study the tests of position invariance of the complex motions seemed to produce both a global effect and a local effect. Such a position invariance test could be also applied to the contingent aftereffect paradigm.

\section{Summary and Conclusions}

We have shown that adaptation to a complex motion produces threshold elevation for patterns undergoing a similar motion and an aftereffect resembling the motion "opposite" to that just observed. These effects were apparent even when the test pattern was positioned so as to be noncoincidental to the center of the adapting flow pattern, suggesting a degree of position invariance. Adapting to spiralling stimuli produces results that are very similar to adapting to curl or div patterns. We suggest our results can be explained by postulating the existence of detectors that are tuned for a variety of complex motions and show a degree of position invariance. Cells of area MST of the macaque appear to exhibit similar properties.

\section{METHODS}

\section{Stimuli}

In designing the stimuli our major concern was to make sure we eliminated any cues to the global motion that might be transmitted by nonmotion or local motion cues. We shall first provide a description of our stimuli and then explain how our manipulations eliminated these cues, and our rational behind these decisions. Our stimuli were constructed according to the same principles as those used to study MSTd cells in the study by Graziano et al. (1994).

Stimuli consisted of elements randomly distributed within an area (this area was conceptual rather than physical, i.e., there were no boundaries explicit to the observer). The screen was bright (luminance $30 \mathrm{~cd} / \mathrm{m}^{2}$ ), whereas each element of the pattern was a dark (luminance $\left.1 \mathrm{~cd} / \mathrm{m}^{2}\right)$ square of side $0.5 \mathrm{~mm}(0.05 \mathrm{deg})$. For most stimuli (termed the large stimuli) the conceptual area was a circle of radius $5 \mathrm{~cm}(5 \mathrm{deg})$ and contained 200 dots (density $=2.55$ dots $/ \mathrm{deg}^{2}$ ), for the small test patterns in the position invariance tests the area was a circle of radius $2.5 \mathrm{~cm}$ (2.5 deg) and contained 50 dots (density $=2.55$ dots $/ \mathrm{deg}^{2}$ ). Stimuli were presented on screen being refreshed at $60 \mathrm{~Hz}$, hence each movie was updated at every $16.6 \mathrm{msec}$. Movie sequences were calculated off-line and stored in memory to be displayed at the appropriate time. Test sequences consisted of 30 frames ( $0.5 \mathrm{sec})$, whereas adaptation sequences consisted of 60 frames $(1.0 \mathrm{sec})$. As we required longer adaptation periods this $1 \mathrm{sec}$ sequence was continually looped through to produce the required times (see Procedures). This gave a slight twitch in the motion sequence.

Optic flow patterns were produced by displacing dots from frame to frame. Dots were given a speed and direction according to their initial position in the stimulus and the type of motion being simulated (expansions/contractions, rotations, and spirals). That dot would then undergo this motion for four displacements (i.e., each dot existed for $83 \mathrm{msec}$ ) before being destroyed and replotted elsewhere on the screen and given speed and direction components appropriate to its new position. Dots were destroyed asynchronously to avoid any coherent flicker. The speed of our dots was such that each dot (no matter which motion it was undergoing) was $2 r \mathrm{deg} / \mathrm{sec}$ where $r$ is its distance (in $\mathrm{cm}$ ) from the center of the pattern. This speed is considerably quicker than that used by Graziano et al. (1994) due to sampling difficulties. Dots near the center of the field move very slowly and, inevitably, on a screen of finite pixel size, this means that the movement of some dots was probabilistic. We therefore used this quicker speed to reduce this number while trying to keep as close to the conditions of Graziano et al. as possible.

Looming stimuli can often produce a change in luminance, the size of pattern elements or density may change, and/or the size of the stimulus may change. Such cues were eliminated from our stimuli. The limited lifetime of our elements meant that the density remains static across both spatial and temporal dimensions and pattern size cues are drastically reduced. Elements remain the same size and together this means that luminance cues are eliminated. We also eliminated some "local motion" related cues, namely those due to accelerations (i.e., elements in an expanding field would normally be increasing in speed as they move away from the center of expansion, elements in a rotating field would normally be changing direction as they move), by having each element move in a straight line at constant speed for its brief lifetime. This possibly counterintuitive manipulation was performed so that subjects required global cues to detect the flow motion. If we had not done so it could be possible that subjects could simply spot a single curved trajectory of an element (or an accelerating element) and surmise the correct motion without any perception of global motion. As all these factors are normally associated with complex motions in the real world it is clear that our stimuli are an impoverished version of the "real thing" and contain many cues that actually contradict the percept (e.g., a looming surface) that we are simulating. Nevertheless stimuli constructed according to the same rules are known to drive cells of area MSTd (Graziano et al., 1994), and Tanaka et al. (1989) provided evidence that it is the global dis- 
tribution of direction that appears to be the most critical factor in driving these MST cells (for psychophysical evidence for the importance of direction see De Bruyn \& Orban, 1990).

In many of our tests we degraded this motion signal so we could measure thresholds. One method is to allow only a certain percentage of the dots to undergo the flow motion, while assigning the rest to be "noise." This general method has become a popular way of measuring thresholds for both linear motion (Newsome \& Paré, 1988; Snowden \& Braddick, 1989), including those after adaptation (Blake \& Hiris, 1993; Raymond, 1993), and more complex motions (Edwards \& Badcock, 1993). However, the way in which noise is defined varies from study to study. This may or may not be an issue for linear motions, but could play an important role for complex motions as employed in the present study. The most general method has been to simply replot "noise" elements randomly on the screen. This, however, then means that the speed distribution of the noise elements is different to the signal elements. In the case of complex motion where the speed profile changes across the stimulus, this could well provide strong cues (e.g., the mere presence of a slowly moving element near the center of the pattern would indicate the presence of the flow field). We therefore produce noise in a way that maintains the same speed profile for the noise elements as for the signal elements. This was done by assigning a random direction for the noise dots while keeping the same speed as would be assigned to a signal dot in this location. Examples of these transformations are seen in Figure 11. One consequence of this is that for $0 \%$ signal (i.e., all noise dots) the stimulus was exactly the same whether it had notionally been derived from an expansion, rotation or spiral motion-each dot had a speed of $2 r$ and a random direction. Again this means that there is nothing but the global motion cues available for our subjects to perform the task.

\section{Procedures}

Subjects were seated $57 \mathrm{~cm}$ from the screen with a patch covering their left eye. The general procedure was explained to the subject. They were then shown examples of the type of flow field they were going to have to detect; all possible patterns (see below) were shown four times at two correlation levels ( 80 and 40\%). Following this subjects were given a short $(10 \mathrm{~min})$ practice run to familiarize themselves with the experimental technique. The importance of keeping strict fixation (especially for the position invariance tests) was explained to the subjects and they were reminded of this at the beginning of each session and several times during a session.

Our general procedure consisted of a preadapting period of $60 \mathrm{sec}$ where the subject viewed the selected adaptation pattern. There then followed a number of top-up/test trials. The top-up adaptation was presented for $4 \mathrm{sec}$ and then after a $0.5 \mathrm{sec}$ blank a trial occurred. Each trial consisted of the presentation of two stimuli separated by $0.5 \mathrm{sec}$. One of these stimuli was always at the $0 \%$ signal level (which we term the standard pattern) while the other had some percentage of the dots undergoing a specified flow (which we term the test pattern).

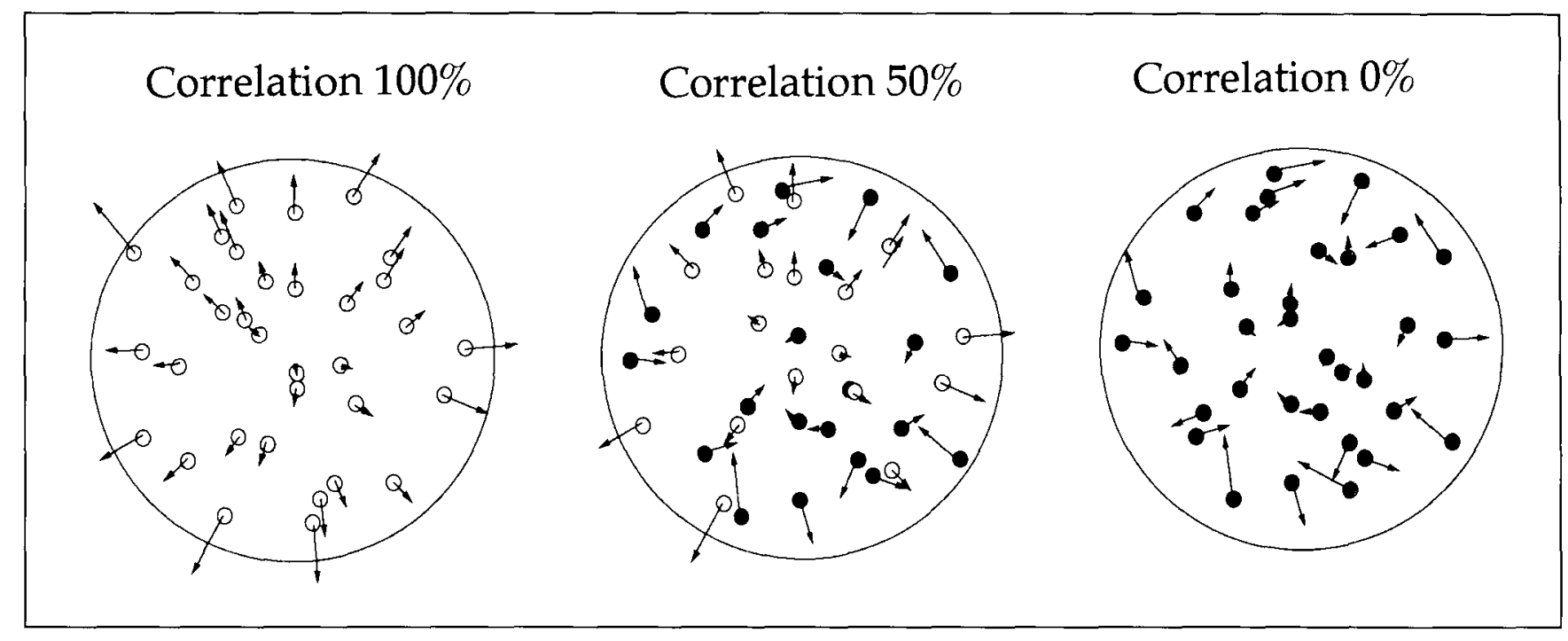

Figure 11. Schematic representation of the stimuli used. In the left section all pattern elements have a direction (arrow direction) and speed (arrow length) commensurate with a surface approaching the observer. As all the elements move in this fashion they may all be regarded as signal and this is termed $100 \%$ correlation. In the right panel the elements still have speeds that are appropriate to a surface moving toward the observer, however, the direction of each element is now random. As the signal has been destroyed in all dots we call this $0 \%$ correlation. In the middle panel half the dots are signal and half move randomly and this is termed $50 \%$ correlation. Note that the movement for noise elements will be the same whether the signal dots were divs, curls, or spirals. 
The order of presentation was randomized from trial to trial and the subject made a two-interval forced choice as to which interval had the test pattern. No feedback was given. Following the response via a button press the next top-up period immediately began. Note that the subject was not required to specify which type of flow field had occurred.

For experiment 1 (position invariance tests) we adapted to a large flow field and tested at two possible positions, one immediately below the fixation point and one immediately above it (see Fig. 2A). On each trial each of the intervals could be either above or below the fixation point (this was to ensure that at no point could the subject anticipate where the pattern would occur). All four possible test patterns [2 positions (upper/lower) by 2 types (expansion/contraction in some experiments and clockwise/anticlockwise in other experiments)] were presented at four signal levels (which levels were determined on pilot trials) thought to span where threshold would be. In total 50 trials were gathered for each of the four stimulus types and the four correlation levels making a total of 800 trials. This would have taken approximately $1.5 \mathrm{hr}$, which was thought far too taxing for a subject who was required to keep strict fixation. We therefore split the experiment into 5 sessions with 10 trials of each possible pattern being performed in each block. All together each subject performed six experiments.

1. Adapt to blank screen test expansion/contractions (baseline)

2. Adapt to expansion test expansion/contractions

3. Adapt to contraction test expansion/contractions

4. Adapt to blank screen test clockwise/anticlockwise rotations (baseline)

5. Adapt to clockwise test clockwise/anticlockwise rotations

6. Adapt to anticlockwise test clockwise/anticlockwise rotations

Two subjects completed this full regime (EJ and RJS).

In the second set of experiments (spiral tests) we adapted to a large flow field and tested with the same size field. In total eight types of test pattern were presented. The patterns are described by the coordinate system shown if Figure 1. If we arbitrarily call the 12 o'clock position $0^{\circ}$ and proceed clockwise our stimuli were $0^{\circ}$ (expansion), $45^{\circ}$ (spiral-expand and clockwise), $90^{\circ}$ (clockwise rotation), $135^{\circ}, 180^{\circ}, 225^{\circ}, 270^{\circ}$, and $315^{\circ}$. Each of these patterns was presented at 4-6 signal levels (again determined in pilot experiments). Again the type of trial and its signal level were randomly chosen for each trial from this set and the subject could not anticipate its type. Fifty trials were run for each trial type (8) and signal level making (at least 4) making at least 1600 trials. This was also therefore run in blocks of 10 trials per condition.
Two subjects (RJS and HA) ran three experiments each.

1. Adapt to blank screen (baseline)

2. Adapt to a cardinal direction $\left(90^{\circ}\right.$ for RJS, $180^{\circ}$ for HA)

3. Adapt to a noncardinal direction $\left(135^{\circ}\right.$ for RJS, $315^{\circ}$ for $\mathrm{HA}$ )

One further subject (MD) ran five experiments (one baseline, two cardinal adaptation directions, and two noncardinal ones). One additional subject (EJ) was run on the baseline condition as described above and another (RM) on a modified version that also included directions at the middle of those described above (i.e., $22.5^{\circ}, 67.5^{\circ}$, etc.).

In addition to the formal experiments described above a number of informal observations were made. The major aim of these observations was simply to observe what might be thought of as the conventional aftereffect of adaptation. Hence we adapted to a moving stimulus and tested by presenting a stationary test patch. The same adaptation regime described above (adapt $1 \mathrm{~min}$, top-up for $4 \mathrm{sec}$, etc.) was used with just a single test interval of $0.5 \mathrm{sec}$. In the test interval various different types of pattern could be presented. The test patterns normally consisted of a pattern of stationary dots (similar to conventional test patterns save that each element still lived for only $83 \mathrm{msec}$ ). The subject simply reported on the perceived aftereffects after each test interval. No measurements were taken to quantify these aftereffects. Various observers and casual visitors to the laboratory and office have been subjected to these informal demonstrations.

\section{Subjects}

A total of five subjects took part in the formal experiments. RJS (male, aged 28-30 at the time of these experiments) is one of the authors and ran all conditions in all experiments as well as extensive pilot studies and is an experienced psychophysical observer. EJ, HA, MD, and RM (females, ages 20-32) were paid naive observers. All observers wore their normal reading glasses if this was appropriate.

\section{Analyses}

For each stimulus type for each observer we had a raw score of the number of correct responses out of 50 . From this we obtained psychometric functions of percent correct against percent signal on linear axes, which were fit by probit analysis to obtain the estimated $75 \%$ correct point (which we will term threshold signal) and a measure of standard error. The standard error of each measurement was typically on the order of $10 \%$ of the threshold. It may, or may not, be more appropriate to fit 
percentage correct to the logarithm of percentage signal. Our data are insufficient to decide this point-but this would have no bearing on the conclusions we wish to draw from this study.

\section{APPENDIX}

Our simulation consisted of a set of Gaussian filters whose response $\left(\boldsymbol{R}_{\mathrm{g}}\right)$ was of the form

$$
R_{\mathrm{g}}=\exp \left\{-0.5\left[(\theta-\omega)^{2} / \sigma^{2}\right]\right\}
$$

where $\theta$ is the angle that the function is centered, $\omega$ is the test angle, and $\sigma$ is the standard deviation (bandwidth) of the function. Gaussian profiles were used as they are specified by a small number of parameters $(\theta$ and $\sigma$ ) and have previously been fit to results from individual cells responding to optic flow patterns (Graziano et al., 1994). The output of each filter $\left(R_{\mathrm{i}}\right)$ was then scaled according to the test correlation (in percent) according to the equation:

$$
R_{\mathrm{i}}=R_{\mathrm{g}} \kappa \phi
$$

where $\phi$ is the correlation of the stimuli and $\kappa$ is a scaling factor. Before adaptation $\kappa$ was set to a value of 1.0 for all filters. We used a linear function, rather than the more popular Naka-Rushton type sigmoidal function, as it has been shown that the response of area MT cells to changes in stimulus correlation is approximately linear (Britten, Shadlen, Newsome, \& Movshon, 1993). Though there are no published data as yet on the response of area MST cells to such stimuli, it seems likely that their behavior will be similar.

Initially, the response of each filter was calculated for a correlation of $1 \%$ in the test direction. If any filter gave a response above a criterion value then this level of correlation was regarded as threshold. If no filter reached this criterion the test correlation was increased in steps of $0.1 \%$ until some filter reached the criterion level. We chose a criterion level of 10 units, as this could be reached by $10 \%$ correlation levels for some test directions and was approximately the sensitivity of the human observers. Summation across filters and probability summation were not simulated.

The response in each filter to the adapting stimulus was calculated in the same manner as above. We then simulated adaptation by changing the factor $\kappa$ in each filter according to the rule

$$
\kappa_{\text {new }}=\kappa_{\text {old }}-R_{\mathfrak{j}} / 150
$$

As the maximum response from a filter is 100 it was possible that $\kappa$ could change from a value of 1 to a value of 0.33 . This adaptation factor was arbitrary and has no influence on the results over a wide range of values. Thresholds for the test stimuli were then calculated with the new $\kappa$ values.

For the simulations using only four filters their peaks $(\theta)$ were at $0,90,180$, and $270^{\circ}$. Examples of simulations with bandwidths $(\sigma)$ of $70^{\circ}$ are shown in Figure 10. For the multifilter model 18 filters were used spaced $20^{\circ}$ apart. This number and spacing were again arbitrary and we suspect could be changed without affecting the results markedly. We found a 12 filter model gave very similar results. The bandwidth $(\sigma)$ was chosen to be $40^{\circ}$ to be as similar as possible to those reported from MST cells (Graziano et al., 1994). Results of this simulation are also shown in Figure 10.

\section{Acknowledgment}

RJS is supported by SERC Grant GR/H52375.

Reprint requests should be sent to Dr. Robert J.Snowden, Vision Research Unit, School of Psychology, University of Wales College of Cardiff, Cardiff CF1 3YG, U.K.

\section{Notes}

1. For some possible criticisms of Regan and colleagues evidence for div and curl detectors see Simpson (1992).

2. British readers may have the pleasure of the spiralling aftereffect several times a week if they desire. The opening credits to the popular soap opera "EastEnders" shows a downward view from an imaginary aircraft that is spiralling away from London. The ground below is therefore spiralling $\left(135^{\circ}\right.$ on the current coordinate system). Near the end of the credits this aircraft stops and the east end of London may be seen to undergo an expansion and anticlockwise rotation.

3. In a subsidiary experiment thresholds for expansions and contractions were measured in 14 naive subjects. Twelve of 14 were significantly more sensitive to contraction than expansion while the other 2 showed no significant differences.

\section{REFERENCES}

Addams, R. (1834). An account of a peculiar optical phaenomenon seen after having looked at a moving body etc. London and Edinburgh Pbilosopbical Magazine and Journal of Science, 3rd series, 5, 373-374.

Albright, T. D. (1984). Direction and orientation selectivity of neurons in visual area MT of the macaque.Journal of Neuroplysiology, 52, 1106-1130.

Albright, T. D. (1989). Centrifugal directional bias in the middle temporal visual area (MT) of the macaque. Visual Neuroscience, 2, 177-188.

Ball, K., \& Sekuler, R. (1980). Human vision favors centrifugal motion. Perception, 9, 317-325.

Blake, R., \& Hiris, E. (1993). Another means for measuring the motion aftereffect. Vision Research, 33, 1589-1592.

Bonnet, C. (1982). Thresholds of motion perception. In A. H. Wertheim, W. A. Wagenaar, \& H. W. Leibowitz (Eds.), Tutorials on motion perception. New York: Plenum Press.

Bonnet, C., \& Pouthas, V. (1972). Interactions between spatial and kinetic dimensions in the movement aftereffect. Perception and Psychophysics, 12, 193-200.

Britten, K. H., Shadlen, M. N., Newsome, W. T., \& Movshon, J. A. (1993). Responses of neurons in macaque MT to sto chastic motion signals. Visual Neuroscience, 10, 11571169.

Broerse, J., Shaw, C., Dodwell, P., \& Muir, D. (1994). Colored aftereffects contingent on global transformations? Spatial Vision, 8, 95-117. 
Cavanagh, P., \& Favreau, O. E. (1980). Motion aftereffect: A global mechanism for the perception of rotation. Perception, 9, 175-182.

Culham, J. C., \& Cavanagh, P. (1995). Motion aftereffects of attentive tracking are rotation-specific but independent of position. Investigative Opbthalmology and Visual Science Supplement, 36, 857.

Cumming, B. (1994). Motion-in-depth. In A. T. Smith \& R. J. Snowden (Eds.), The visual detection of motion. San Diego: Academic Press

De Bruyn, B., \& Orban, G. A. (1990). The role of direction information in the perception of geometric flow components. Perception and Psychopbysics, 47, 433-438.

De Bruyn, B., \& Orban, G. A. (1993). Segregation of spatially superimposed optic flow components. Journal of Experimental Psychology; Human Perception and Performance 19, 1014-1027.

Duffy, C. J., \& Wurtz, R. H. (1991a). Sensitivity of MST neurons to optic flow stimuli. I. A continuum of response selectivity to large-field stimuli. Journal of Neuropbysiology, 65, 1329-1345.

Duffy, C. J., \& Wurtz, R. H. (1991b). Sensitivity of MST neurons to optic flow stimuli. II. Mechanisms of response selectivity revealed by small-field stimuli.Journal of Neuropbysiology, 65, 1346-1359.

Edwards, M., \& Badcock, D. (1993). Asymmetries in the sensitivity to motion in depth: A centripetal bias. Perception, 22, 1013-1023.

Emerson, V. F., Humphrey, G. K., \& Dodwell, P. C. (1985). Colored aftereffects contingent on patterns generated by Lie transform groups. Perception and Psychopbysics, 37, 155162

Freeman, T. C. A., \& Harris, M. G. (1992). Human sensitivity to expanding and rotating motion: Effects of complementary masking and directional structure. Vision Research, 32, 81-87.

Gibson, J. J. (1950). The perception of the visual world. Boston: Houghton Mifflin.

Goldstein, A. G. (1958). On the after-effects of the waterfall and spiral illusions. American Journal of Psychology, 71, 608-609.

Graziano, M. S. A., Andersen, R. A., \& Snowden, R. J. (1994). Tuning of MST neurons to spiral stimuli. Journal of Neuroscience, $14,54-67$.

Hershenson, M. (1993). Linear and rotation motion aftereffects as a function of inspection duration. Vision Research, 33, 1913-1919.

Holland, H. C. (1965). The spiral aftereffect. Oxford: Pergamon.

Koenderink, J. J. (1986). Optic flow. Vision Research, 26, 161180.

Koenderink, J. J., \& van Doorn, A. J. (1975). Invariant properties of the motion parallax field due to the movement of rigid bodies relative to an observer. Optica Acta, 22, 773791

Lee, D. (1976). A theory of visual control of braking based on information about time to collision. Perception, 5, 437459.

Legge, G. E., \& Campbell, F. W. (1981). Displacement detection in human vision. Vision Research, 21, 205-214.

Longuet-Higgins, H. C., \& Prazdny, K. (1980). The interpretation of moving retinal images. Proceedings of the Royal Society, London, 8 208, 385-387.

Mather, G. (1980). The movement aftereffect and a distribution-shift model for coding the direction of visual movement. Perception, 9, 379-382.

Maunsell, J. H. R., \& van Essen, D. C. (1983). Functional properties of neurons in middle temporal visual area of the ma- caque monkey. I. Selectivity for stimulus direction, speed, and orientation. Journal of Neuropbysiology, 49, 11271147.

Maunsell, J. H. R., \& van Essen, D. C. (1987). Topographic organization of the middle temporal visual area in the macaque monkey: Representational biases and the relationship to callosal connections and boundaries. Journal of Comparative Neurology, 266, 535-555.

McCollough, C. (1994). Do McCollough effects provide evidence of global pattern-processes? Investigative Opbthalmology and Visual Science Supplement, 35, 1489.

Milne, A. B., \& Snowden, R. J. (1993). Is there anything special about expansion and rotational flow fields? Perception, 22, 95A.

Mollon, J. D. (1974). After-effects and the brain. New Scientist, 61, 479-482.

Morrone, M. C., Burr, D. C., \& Vaina, L. M. (1995). Two stages of visual processing for radial and circular motion. Nature (London), 376, 507-509.

Nakayama, K., \& Tyler, C. W. (1981). Psychophysical isolation of movement sensitivity by removal of familiar position cues. Vision Research, 21, 427-433.

Newsome, W. T., \& Paré, E. B. (1988). A selective impairment of motion perception following lesions of the middle temporal visual area (MT). Journal of Neuroscience, 8, 22012211

Orban, G. A., Lagae, L., Raiguel, S., Xiao, D., \& Maes, H. (1995). The speed tuning of medial superior temporal (MST) cell responses to optic-flow components. Perception, 24, 269285.

Plateau, J. (1850). Vierte Notiz uber eine neue sonderbare Anwendung des Verweilens der Eindrucke auf der Netzhaut. Poggendorff's Annalen, 80, 287-292.

Raymond, J. (1993). Movement direction analysers: Independence and bandwidth. Vision Research, 33, 767-775.

Raymond, J. E. (1994). Directional anisotropy of motion sensitivity across the visual field. Vision Research, 34, 10291038.

Regan, D., \& Beverley, K. I. (1978a). Looming detectors in the human visual pathway. Vision Research, 18, 415-421.

Regan, D., \& Beverley, K. (1978b). Illusory motion in depth: After-effect of adaptation to changing size. Vision Research, 18, 209-212.

Regan, D., \& Beverley, K. I. (1985). Visual responses to vorticity and the neural analysis of optic flow. Journal of the $O p$ tical Society of America A, 2, 280-283.

Saito, H.-A., Yukie, M., Tanaka, K., Hikosaka, K., Fukada, Y., \& Iwai, E. (1986). Integration of direction signals of image motion in the superior temporal sulcus of the macaque monkey. Journal of Neuroscience, 6, 145-157.

Siegel, R. M., \& Andersen, R. A. (1986). Motion perceptual deficits following ibotenic acid lesions of the middle temporal area in the behaving rhesus monkey. Society for Neuroscience Abstracts, 12, 1183.

Simpson, J. I. (1984). The accessory optic system. Annual Review of Neuroscience, 7, 13-41.

Simpson, W. A. (1992). Optic flow and depth perception. Spatial Vision, 7, 35-75

Snowden, R. J. (1992). Sensitivity to relative and absolute motion. Perception, 21, 563-568.

Snowden, R. J. (1994). Motion processing in the primate cerebral cortex. In A. T. Smith \& R. J. Snowden (Eds.), Visual detection of motion. San Diego: Academic Press.

Snowden, R. J., \& Braddick, O. J. (1989). The combination of motion signals over time. Vision Research, 29, 1621-1630.

Snowden, R. J., \& Milne, A. B. (1994). Evidence for the existence of mechanisms tuned for complex motions in the 
human visual system. Investigative Opbthalmology and Visual Science Supplement, 35, 1726.

Snowden, R. J., Treue, S., \& Andersen, R. A. (1992). The response of neurons in area V1 and MT of the alert rhesus monkey to moving random dot patterns. Experimental Brain Research, 88, 389-400.

Tanaka, K., Fukada, Y., \& Saito, H. (1989). Underlying mechanisms of the response specificity of the expansion/contraction and rotation cells in the dorsal part of the medial superior temporal area of the macaque monkey. Journal of Neuropbysiology, 62, 642-656.

Tanaka, K., Hikosaka, K., Saito, H.-A., Yukie, M., Fukada, Y., \& Iwai, E. (1986). Analysis of local and wide-field movements in the superior temporal visual areas of the macaque monkey. Journal of Neuroscience, 6, 134-144.

Tanaka, K., \& Saito, H. (1989). Analysis of motion of the vis- ual field by direction, expansion/contraction, and rotation cells clustered in the dorsal part of the medial superior temporal area of the macaque monkey. Journal of Neuropbysiology, 62, 626-641.

Tyler, C. W., \& Torres, J. (1972). Frequency response characteristics for sinusoidal movement in the fovea and periphery. Perception and Psychopbysics, 12, 232-236.

Weisstein, N., Maguire, W., \& Berbaum, K. (1977). A phantommotion aftereffect. Science, 198, 955-958.

Wohlgemuth, A. (1911). On the aftereffect of seen motion. British Journal of Psycbology, 1, 1-117.

Zeki, S. M. (1980). The response properties of cells in the middle temporal area (area MT) of owl monkey visual cortex. Proceedings of the Royal Society of London B, 207, 239-248. 